\title{
A Canadian Take on the International Patient Engagement Revolution
}

Vincent Dumez and Antoine Boivin

$\mathrm{F}$ ive years ago, in the UK, the $B M J$ argued that a "patient revolution" was afoot in the healthcare system (Richards et al. 2013). The same year, in the US, Health Affairs called patient engagement the "blockbuster drug of the century." Over a 10-year period, the number of international publications on patient engagement tripled (Boote et al. 2012). Canada is no exception to this international trend, and a growing number of health professionals and organizations are engaging patients, families and citizens as partners in the care improvement journey.

This Special Issue of Healthcare Quarterly aims to provide an overview of patient and family engagement in healthcare system improvement. It brings together experts (patients, clinicians, researchers, policy makers) from across the country tasked with sharing their experiential learning regarding engagement in the Canadian healthcare system. A team of experts from the Netherlands was added to this group of authors to provide insights into European developments in this area.

The authors who were invited to contribute to this Special Issue are all pioneers and leaders in patient, family and public partnerships. Their contributions to this field as academics and agents of change are made from both the perspective of observers and the standpoint of key influencers in the development of patient, family and public engagement across the country. They are undoubtedly best positioned to provide an assessment of the current situation and to help us better understand the next steps. Most of the articles include patients as co-authors, together with clinicians, managers and researchers. Similarly, this Special Issue is co-edited by a patient and a clinician-researcher.

\section{Supporting Engagement-Capable Environments in Canada}

The opening article of this issue, "Supporting Patient and Family Engagement for Healthcare Improvement: Reflections on 'Engagement-Capable Environments' in Pan-Canadian Learning Collaboratives," was written by Carol Fancott, G. Ross Baker and Maria Judd, along with patient partners Anya Humphrey and Angela Morin (Fancott et al. 2018). It focuses on the role played by the Canadian Foundation for Healthcare Improvement (CFHI) in recent years to help develop patient engagement capacity in Canadian healthcare organizations. The foundation also made a bold choice in encouraging its teams to embed shared leadership with patients and families in the projects it funds in order to promote a co-design and co-build approach throughout the entire process. Building on the notion of "engagement-capable environments" found throughout this Special Issue, the authors describe how a national organization can foster engagement at the organizational level.

\section{Building Organizational Capacity for Patient and Family Engagement}

The next articles in this Special Issue focus on the three main pillars of engagement-capable environments: leadership, readiness of staff/teams to engage and the role of engaged patients. 
"The Leadership and Organizational Context Required to Support Patient Partnerships" focuses on the structures and cultural transformations brought about by leaders of change as champions of patient engagement. It was written by a trio made up of Patricia O'Connor, a former director of nursing, Mario Di Carlo, a patient partner involved in multiple Canadian patient and family engagement initiatives, and Jean-Lucien Rouleau, co-founder of the Patient Partnership Program while he was dean of the Faculty of Medicine at the Université de Montréal (O’Connor et al. 2018).

"Development of Patient-Inclusive Teams: Toward a Structured Methodology" (Pomey et al. 2018) focuses on supportive conditions for the integration of patients and families in professional healthcare teams. It was written by a diverse Quebec-based team made up of researchers, professors and students from the Université de Montréal, as well as experienced decision-makers and patients from the Quebec health and social services system: Drs. Marie-Pascale Pomey, Paule Lebel, Nathalie Clavel, Catherine Neault, Benoît Tétreault and Anna-Paulina Ewalds Mulliez, as well as patients Édith Morin and Mireille Morin.

"Patient Roles in Engagement-Capable Environments: Multiple Perspectives" (Rowland et al. 2018) was written by the trio of Paula Rowland, from the University of Toronto, and the patient-professional dyad currently leading the implementation of Patient Partnerships at Accreditation Canada: Claudia Houle and Mireille Brosseau. Rowland, Houle and Brosseau speak to the complexity of roles played by patients in health organizations, beyond their official "job description."

Our European collaborators provide an international perspective on engagement practices (Faber et al. 2018) in "Implementation of Patient Engagement in the Netherlands: A Stimulating Environment within a Large Academic Medical Centre." Marjan Faber and the team of Thomas Vijn, Marja Jillissen, David Grim and Jan Kremer from Radboud university medical center Nijmegen describe the evolution of patient and family engagement activities at a large teaching hospital in the Netherlands, one of the most successful health systems in the OECD. Radboud is part of a core group of teaching hospitals in continental Europe that focus on innovative patient and family engagement strategies. Despite some contextual differences, this European example highlighting organizational dynamics, transformation levers and resistance resonates with several Canadian examples.

\section{Integrating Research and Evaluation}

The last two original contributions of this issue focus on integrating research and evaluation in patient engagement activities with a view to improving care.

"Bringing Together Research and Quality Improvement: The Saskatchewan Approach" (Teare et al. 2018) describes how support structures for patient engagement in research and quality of care can be integrated at the provincial level. It was written by the duo who took the lead on deploying patient and family engagement in quality improvement across Saskatchewan, Gary Teare, Malori Keller, as well as patient advisor Dale Hall.

Julia Abelson and two postdoctoral fellows, Ania Syrowatka and Julia Bidonde, joined a patient partner with extensive experience in quality improvement projects, Anya Humphrey, and CFHI's vice-president of programs, Maria Judd, to write "Evaluating Patient, Family and Public Engagement in Health Services Improvement and System Redesign" (Abelson et al. 2018). They offer up ideas on how to integrate evaluation into engagement activities by clarifying its various roles and introducing examples of available assessment tools for practitioners.

\section{Future Outlook}

"The Capacity for Patient Engagement: What Patient Experiences Tell Us About What's Ahead" (Canfield 2018) was written by Carolyn Canfield, a patient advisor with extensive experience in various Canadian and international patient and family engagement initiatives. She is the co-founder of the budding Patient Advisors Network (PAN), which brings together experienced and engaged patients across Canada. She shares her reflections on barriers to engagement and the self-selection of engaged patients, as well as possible solutions to support the development of a wider and more diversified community of engaged patients.

The editorial team's summary article (Boivin et al. 2018), "Growing a Healthy Ecosystem for Patient and Citizen Partnerships," provides an ecosystem perspective on engagement, including key individual, organizational and systemic components that support reciprocal and effective relationships with patients and citizens.

Ultimately, the articles in this Special Issue provide a 360-degree view of our country's level of maturity with regard to patient and family engagement in healthcare improvement, while describing the challenges that lie ahead for scaling up and sustaining what many call a revolution. HQ

\section{Acknowledgements}

The editors would especially like to thank Carol Fancott for the quality of her support throughout all stages of the design and editing process of this Special Issue.

\section{References}

Abelson, J., A. Humphrey, A. Syrowatka, J. Bidonde and M. Judd. 2018. "Evaluating Patient, Family and Public Engagement in Health Services Improvement and System Redesign." Healthcare Quarterly 21(Special Issue): 61-67. doi:10.12927/hcq.2018.25636.

Boivin, A., V. Dumez, C. Fancott and A. L'Espérance. 2018. "Growing a Healthy Ecosystem for Patient and Citizen Partnerships." Healthcare Quarterly 21(Special Issue): 73-82. doi:10.12927/hcq.2018.25634. 
Boote, J., R. Wong and A. Booth. 2012. "Talking the Talk or Walking the Walk?" A Bibliometric Review of the Literature on Public Involvement in Health Research Published between 1995 and 2009." Health Expectations 18: 44-57. doi:10.1111/hex.12007.

Canfield, C. 2018. “The Capacity for Patient Engagement: What Patient Experiences Tell Us About What's Ahead." Healthcare Quarterly 21(Special Issue): 68-72. doi:10.12927/hcq.2018.25635.

Faber, M.J., T.W. Vijn, M. Jillissen, D. Grim and J.A.M. Kremer. 2018. "Implementation of Patient Engagement in the Netherlands: A Stimulating Environment within a Large Academic Medical Centre." Healthcare Quarterly 21(Special Issue): 50-55. doi:10.12927/ hcq.2018.25638.

Fancott, C., G.R. Baker, M. Judd, A. Humphrey and A. Morin. 2018. "Supporting Patient and Family Engagement for Healthcare Improvement: Reflections on 'Engagement-Capable Environments' in Pan-Canadian Learning Collaboratives." Healthcare Quarterly 21(Special Issue): 12-30. doi:10.12927/hcq.2018.25642.

O'Connor, P., M. Di Carlo and J.-L. Rouleau. 2018. "The Leadership and Organizational Context Required to Support Patient Partnerships." Healthcare Quarterly 21(Special Issue): 31-37. doi:10.12927/hcq.2018.25641.

Pomey, M.-P., P. Lebel, N. Clavel, É. Morin, M. Morin, C. Neault, B. Tétreault and A.-P. Ewalds Mulliez. 2018. "Development of PatientInclusive Teams: Toward a Structured Methodology." Healthcare Quarterly 21(Special Issue): 38-44. doi:10.12927/hcq.2018.25640.
Richards, T., V.M. Montori, F. Godlee, P. Lapsley and D. Paul. 2013. "Let the Patient Revolution Begin." BMJ 346: f2614. doi:10.1136/ bmj.f2614.

Rowland, P., M. Brosseau and C. Houle. 2018. "Patient Roles in Engagement-Capable Environments: Multiple Perspectives." Healthcare Quarterly 21(Special Issue): 45-49. doi:10.12927/hcq.2018.25639.

Teare, G.F., M. Keller and D. Hall. 2018. "Bringing Together Research and Quality Improvement: The Saskatchewan Approach.” Healthcare Quarterly 21(Special Issue): 56-60. doi:10.12927/hcq.2018.25637.

\section{About the Authors}

Vincent Dumez is the patient guest editor of this Special Issue and co-director, Centre of Excellence on Partnership with Patients and the Public, University of Montreal, Montreal, QC.

Antoine Boivin is the research guest editor of this Special Issue and co-director, Centre of Excellence on Partnership with Patients and the Public, University of Montreal, Montreal, OC.

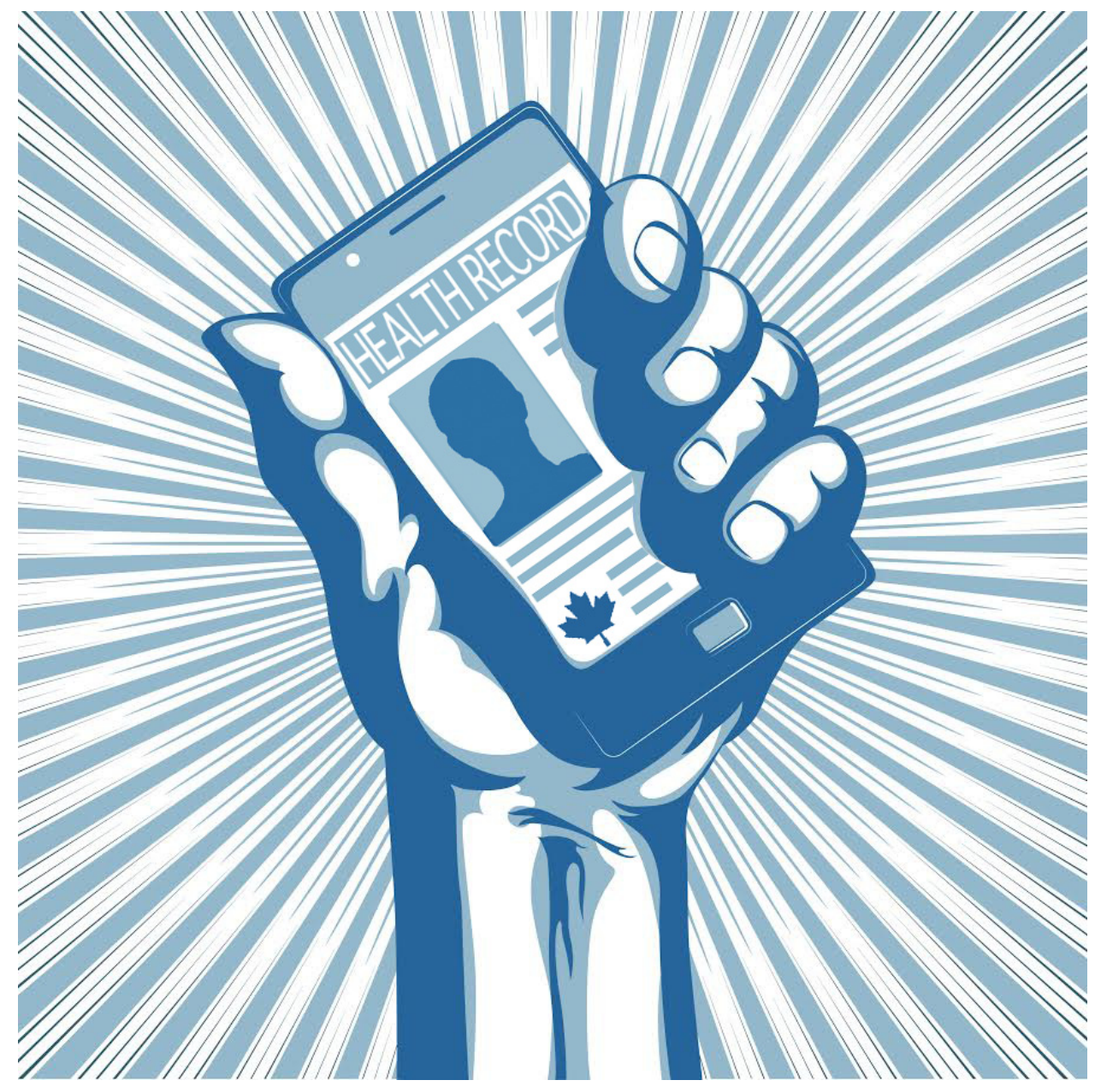




\section{Un portrait canadien de la révolution internationale sur l'engagement des patients}

Vincent Dumez et Antoine Boivin

I ly a 5 ans, le $B M J$ au Royaume-Uni annonçait une « révolution des patients " dans le système de santé (Richards et al. 2013). La même année, le journal Health Affairs aux États-Unis présentait l'engagement des patients comme le " traitement du siècle" (blockbuster drug of the century). Sur une période de 10 ans, le nombre de publications internationales sur l'engagement des patients a été multiplié par trois (Boote et al. 2012). Le Canada n'échappe pas à cette tendance internationale et un nombre croissant de professionnels et d'organisations de santé s'engagent avec les patients, les familles et les citoyens en tant que partenaires dans l'amélioration des soins.

Ce numéro spécial de Healthcare Quarterly vise à dresser un portrait de l'engagement des patients et des familles dans l'amélioration du système de santé au Canada. Il réunit des experts (patients, cliniciens, chercheurs, décideurs) de partout au pays à qui nous avons demandé de partager les apprentissages issus d'expériences pratiques d'engagement dans le système de santé canadien. Une équipe d'experts des Pays-Bas s'ajoute à ces auteurs pour mettre en perspective l'expérience canadienne avec les développements européens dans le domaine.

Les auteurs invités pour écrire dans cette édition spéciale sont tous des pionniers et des leaders du travail en partenariat avec les patients, les familles et le public. Ils ont contribué grandement à ce domaine en tant que concepteurs académiques mais aussi en tant que leaders de changement. Ils sont des observateurs et acteurs clés du développement de l'engagement des patients, des familles et du public au pays. Ce sont certainement aujourd'hui les personnes les mieux placées pour nous proposer un bilan de la situation mais aussi pour nous aider à mieux comprendre les prochaines étapes de développement. Dans la quasi-totalité des articles, des patients travaillant avec des cliniciens et gestionnaires ont été intégrés comme auteurs. De même, le numéro spécial est co-édité par un patient et un clinicien-chercheur.

\section{Soutenir les environnements propices à I'engagement au Canada}

L'article d'ouverture de ce numéro par Fancott, Baker et Judd, avec les partenaires patients Humphrey et Morin (Fancott et al. 2018), présente le rôle que la Fondation Canadienne sur l'amélioration des services de santé a joué dans les dernières années pour soutenir le développement des capacités d'engagement des patients des établissements de santé au Canada. La Fondation a aussi fait le choix courageux d'inciter les collaborateurs des projets qu'elle finance à intégrer un leadership partagé avec les patients et leur famille afin de promouvoir une approche de co-design et co-construction à travers tout le processus de réalisation. Ancré dans le concept « d'environnement propice à l'engagement " (engagement-capable environment) repris à travers ce numéro spécial, les auteurs décrivent comment une organisation nationale peut soutenir les capacités d'engagement d'organisations locales de santé. 


\section{Construire la capacité organisationnelle pour I'engagement des patients et des familles}

Plusieurs articles de cette édition spéciale se concentrent sur les trois principaux piliers des « environnements propices à l'engagement » : le leadership, la préparation du personnel / des équipes à s'engager et le rôle des patients lorsqu'ils s'engagent.

L'article « Leadership and Organizational Context Required to Support Patient Partnerships » met l'accent sur les structures et les transformations culturelles portées par les leaders de changement agissant comme champions de l'engagement des patients. Il est écrit par un trio constitué tout d'abord par une ancienne Directrice des soins infirmiers, Mme Patricia O'Connor, M. Mario Di Carlo, patient partenaire au sein de multiples initiatives d'engagement des patients et des familles au Canada, et le Dr Jean -Lucien Rouleau, co-fondateur du programme partenariat patient alors qu'il était doyen de la Faculté de médecine de l'Université de Montréal (O'Connor et al. 2018).

L'article « Development of Patient-Inclusive Teams: Towards a Structured Methodology» (Pomey et al. 2018) met l'accent sur les conditions propices à l'intégration de patients et membres de la famille au sein des équipes professionnelles des établissements de santé. Il est écrit par une équipe québécoise diversifiée constituée de chercheurs, d'enseignants et d'étudiants de l'Université de Montréal ainsi que de décideurs et patientes expérimentées du système de santé et des services de santé du Québec : Dres Marie-Pascale Pomey, Paule Lebel, Nathalie Clavel, Catherine Neault, Benoît Tétreault, Anna-Paulina Ewalds Mulliez ainsi que les patientes Édith Morin et Mireille Morin.

Larticle « Patient Roles in Engagement-Capable Environments: Multiple Perspectives » (Rowland et al. 2018) est écrit par un trio constitué par Mme Paula Rowland, de l'Université de Toronto, accompagnée par le duo patiente-professionnelle qui assume actuellement le leadership de l'implantation du partenariat patient au sein d'Agrément Canada : Mme Claudia Houle et Mme Mireille Brosseau. Rowland, Houle et Brosseau illustrent la complexité des rôles joués par les patients au sein des organisations de santé, au-delà de leur « description de tâche » officielle.

Nos collaborateurs européens fournissent une perspective internationale des pratiques d'engagement (Faber et al. 2018). Marjan Faber et l'équipe de Vijn, Jillissen, Grim et Kremer de l'hôpital universitaire Radboud Nijmegen décrivent l'évolution des activités d'engagement des patients et des familles au sein d'un centre hospitalier universitaire des Pays-Bas, un des systèmes de santé les plus performants parmi les pays de l'OCDE. Radboud a été parmi les centres hospitaliers en Europe continentale dans le développement d'innovations d'engagement des patients et des familles. Malgré certaines différences contextuelles, cet exemple européen illustre les dynamiques organisationnelles, leviers de transformation et résistances qui résonnent avec plusieurs exemples canadiens.

\section{Intégrer la recherche et l'évaluation}

Les deux dernières contributions originales du numéro se penchent sur l'intégration de la recherche et de l'évaluation dans les activités d'engagement des patients en amélioration des soins.

L'article "Bringing Together Research and Quality Improvement: The Saskatchewan Approach » (Teare et al. 2018) décrit l'intégration de structures de soutien à l'engagement des patients en recherche et en qualité des soins au niveau d'une infrastructure provinciale. Il est écrit par le duo qui a assumé le leadership du déploiement de l'engagement des patients et des familles dans l'amélioration de la qualité en Saskatchewan : Dr Gary Teare, Mme Malori Keller et Dale Hall.

Julia Abelson et deux stagiaires postdoctorales, Mme Ania Syrowatka et Mme Julia Bidonde, se sont jointes à une patiente partenaire expérimentée en projets d'amélioration de la qualité, Mme Anya Humphrey, et la vice-présidente des programmes de la Fondation, Mme Maria Judd, pour écrire l'article "Evaluating Patient, Family and Public Engagement in Health Services Improvement and System Redesign " (Abelson et al. 2018). Elles présentent un portrait sur la façon dont l'évaluation peut s'intégrer aux activités d'engagement, en clarifiant ses différents rôles et présentant des exemples d'outils d'évaluation disponibles pour les praticiens.

\section{Perspectives futures}

Larticle « The Capacity for Patient Engagement: What Patient Experiences Tell Us About What's Ahead » (Canfield 2018) est écrit par Mme Carolyn Canfield, une patiente conseillère (patient advisor) impliquée depuis plusieurs années dans différentes initiatives d'engagement des patients et des familles au Canada. Elle est une des co-fondatrices de l'organisation en émergence Patient Advisors Network (PAN) qui réunit des patients expérimentés et impliqués à l'échelle du Canada. Elle partage ses réflexions sur la problématique des barrières à l'engagement et de l'auto-sélection de patients engagés, de même que des pistes de solution pour soutenir le développement des capacités d'engagement d'une communauté plus large de patients.

L'article synthèse en conclusion de ce numéro spécial, écrit par l'équipe éditoriale (Boivin et al. 2018), « Growing a Healthy Ecosystem for Patient and Citizen Partnership ", présente une perspective écosystémique sur l'engagement, présentant les éléments clés à l'échelle individuelle, organisationnelle et systémique soutenant des relations réciproques et efficaces avec les patients et les citoyens.

Au total, la somme des articles rassemblée dans ce numéro spécial donne une vision à 360 degrés du niveau de maturité de ce qui se passe actuellement dans notre pays tout en exposant les défis à relever pour aller plus loin dans la mise à l'échelle et la pérennité de ce que beaucoup appellent une révolution. HQ 


\section{Remerciements}

Les éditeurs tiennent à remercier tout spécialement Mme Carol Fancott pour la qualité de son soutien à toutes les étapes du processus de conception et d'édition de ce numéro spécial.

\section{Références}

Veuillez vous reporter à la liste dans la version anglaise (pp. 2-3).

\section{Au sujet des auteurs}

Vincent Dumez est le patient guest editor de ce numéro spécial et co-directeur, Centre d'excellence sur le partenariat avec les patients et le public, Université de Montréal, Montréal, QC.

Antoine Boivin est le research guest editor de ce numéro spécial et co-directeur, Centre d'excellence sur le partenariat avec les patients et le public, Université de Montréal, Montréal, QC.

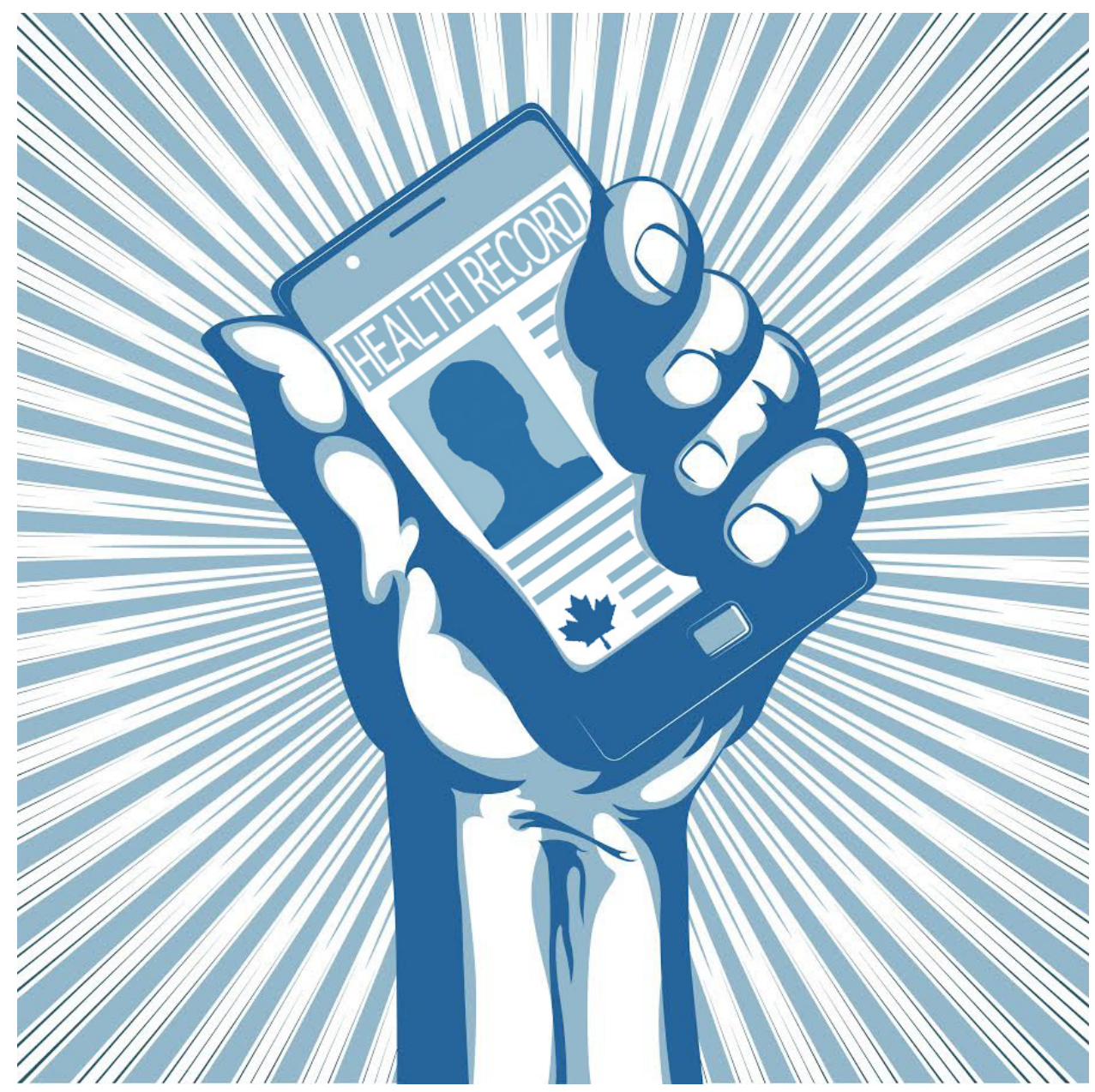

